\title{
Téoros
}

Revue de recherche en tourisme

\section{Géotourisme urbain}

\section{Le cas de Montréal}

\section{Alain Côté, Marie-Claude Joly et Alix Verner}

Volume 28, numéro 2, 2009

URI : https://id.erudit.org/iderudit/1024813ar

DOI : https://doi.org/10.7202/1024813ar

Aller au sommaire du numéro

Éditeur(s)

Université du Québec à Montréal

ISSN

0712-8657 (imprimé)

1923-2705 (numérique)

Découvrir la revue

Citer cette note

Côté, A., Joly, M.-C. \& Verner, A. (2009). Géotourisme urbain : le cas de Montréal. Téoros, 28(2), 97-99. https://doi.org/10.7202/1024813ar d'utilisation que vous pouvez consulter en ligne.

https://apropos.erudit.org/fr/usagers/politique-dutilisation/ 


\section{Géotourisme urbain}

\section{Le cas de Montréal}

\author{
Alain CÔTÉ \\ Maîtrise en gestion et planification du tourisme \\ Université du Québec à Montréal \\ alain_cote_@hotmail.com \\ Marie-Claude JOLY \\ Maîtrise en gestion et planification du tourisme \\ Université du Québec à Montréal \\ m.c.joly@hotmail.com
}

\author{
Alix VERNER \\ Maîtrise en études urbaines \\ Institut national de la recherche scientifique \\ alixverner@hotmail.com
}

\section{Contexte}

Aujourd'hui, les destinations touristiques doivent composer avec une concurrence de plus en plus vive. L'enjeu est encore plus grand pour les destinations urbaines; chaque ville doit mettre en valeur une caractéristique qui la distingue. Depuis octobre 2007, Montréal axe sa stratégie sur le fait d'être la première ville du monde à avoir signé la Charte en géotourisme de la National Geographic Society.

Le géotourisme est un type de tourisme qui sauvegarde et valorise le caractère géographique d'une destination - son environnement, sa culture, son esthétisme, son patrimoine et le bien-être de ses habitants (Center for Sustainable Destinations, 20 août 2009). Il englobe le concept du tourisme durable qui favorise la préservation du site pour les générations futures et la sauvegarde de son caractère particulier. Les principes du géotourisme ont été définis, en 2002, par le Center for Sustainable Destinations (CSD) de la National Geographic.

Une bonne pratique du géotourisme implique que les revenus et autres bénéfices tirés des activités touristiques servent à promouvoir la conservation des lieux.

Afin que les destinations puissent emprunter l'approche du géotourisme, le CSD a rédigé une charte composée de 13 principes : intégrité des lieux, codes internationaux, marché ciblé, marché diversifié, satisfaction des touristes, participation de la communauté, avantages pour la communauté, conservation et valorisation des attraits de la destination, utilisation du territoire, conservation des ressources, planification, interprétation interactive, évaluation.

Dans le cas de Montréal, l'application de ces 13 principes a été l'aboutissement d'un parcours ponctué de débats publics, de recherches, de projets et de processus participatifs au sein d'institutions et de communautés locales. Ces pratiques et ces outils servent à répondre aux besoins des diverses formes du patrimoine, naturel et culturel, bâti dans le contexte urbain d'une ville cosmopolite d'ascendance française aux multiples héritages.
L'adhésion à la Charte en géotourisme implique la reconnaissance collective de cette avancée et un engagement à poursuivre la mise en œuvre des meilleures pratiques en matière de sauvegarde et de valorisation de l'Île-de-Montréal ainsi qu'une satisfaction accrue des touristes et de la communauté environnante. Étant la première destination urbaine au monde à signer cette charte, Montréal entend jouer un rôle de premier plan dans ce sens.

\section{Le projet géotouristique de Montréal}

Trois organismes sont à l'origine de l'adhésion de Montréal à la Charte en géotourisme : le Centre d'excellence sur les destinations touristiques (CED), Tourisme Montréal et Héritage Montréal. Depuis l'adhésion, un comité local a été formé avec la mission de mettre en application les 13 principes de la Charte et de favoriser l'émergence de projets durables qui s'appuieront sur l'ensemble des champs d'expertise de Montréal. Les membres du comité sont issus d'une douzaine d'organismes des secteurs public et privé des milieux de la culture, du tourisme et de l'urbanisme.

Dès le départ, le comité a mobilisé ses efforts pour concevoir un site Web ainsi que la première carte géotouristique en collaboration avec le CSD. Par la réalisation de ce projet, Montréal démontre son soutien aux principes de la Charte et manifeste aux citoyens sa volonté de garder l'esprit ouvert sur une nouvelle façon de gérer le tourisme urbain.

La carte géotouristique est une carte recto verso, de la taille d'une affiche, créée par la National Geographic et le comité local. Elle répertorie les attraits naturels, culturels, historiques et patrimoniaux, ainsi que les expériences locales d'une destination. En contribuant à sauvegarder et à valoriser les atouts de cette région, cette carte participe aux efforts de développement économique de la communauté. La National Geographic Society a fait appel à ses procédés reconnus et à son expertise pour créer la carte géotouristique. L'entreprise a déjà conçu des produits similaires dans d'autres parties du monde. Son 


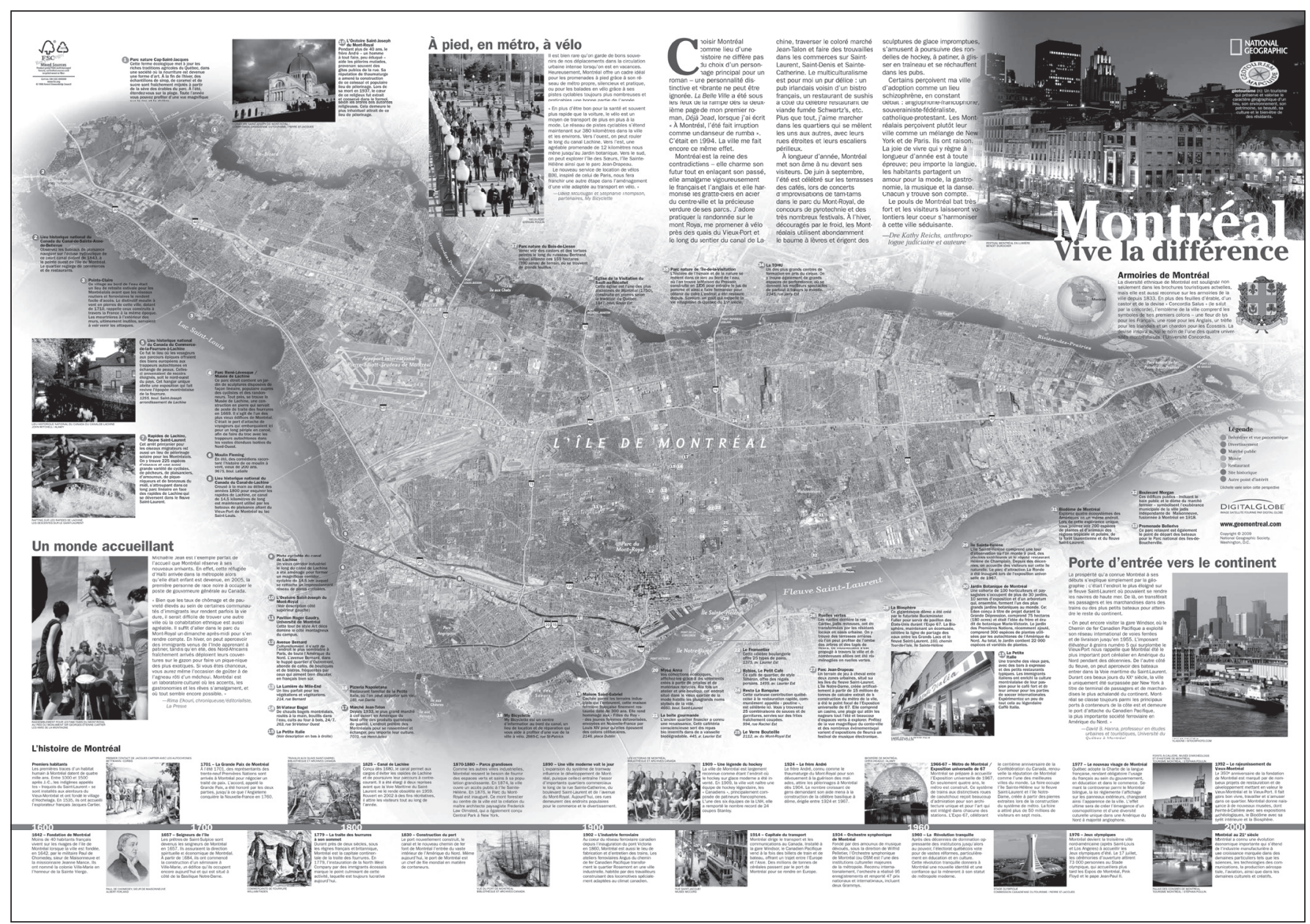

ILLUSTRATION 1 : Carte-guide géotouristique de Montréal (source : Center for Sustainable Destination de la National Geographic Society).

rôle était de valider les sites sélectionnés, puis de les mettre en lumière par des textes, une conception et une impression de qualité supérieure sous forme de carte géotouristique portant le sceau de la National Geographic. Le rôle du comité en géotourisme était de déterminer les limites territoriales de la carte géotouristique, en l'occurrence l'Île-de-Montréal, d'encourager la communauté à participer au processus de la sélection des sites, de veiller à ce que la sélection des sites reflète la diversité de la ville, de planifier la diffusion de la carte géotouristique, de développer un site d'accompagnement et de soutenir d'autres efforts de sensibilisation.

\section{La carte géotouristique}

À ce jour, Montréal est la seule destination urbaine qui a fait l'objet d'une carte-guide parmi la collection existante des dix cartes géotouristiques ${ }^{1}$. Établissant de nouveaux critères pour la conception de la carte, la ville est devenue une référence en la matière. La National Geographic a validé la sélection des sites en se basant sur les 13 principes de la Charte et sur d'autres caractéristiques en matière de développement durable. La carte-guide a été dévoilée le 15 juin 2009 et diffusée localement par Tourisme Montréal dans les centres touristiques et dans les entreprises représentées sur la carte, ainsi qu’à l'échelle internationale par l'intermédiaire des bureaux de Tourisme Montréal à l'étranger. Une image satellite de Montréal apparait au recto de la carte tandis que le verso fournit un plan des rues de Montréal et du Vieux-Montréal, et des suggestions sur les meilleurs endroits en ville en matière de restaurants, boutiques, architecture, musées, promenades. La carte a été publiée en français et en anglais sur du papier recyclé. Elle comporte également une liste descriptive de sites d'intérêt, d'hôtels et de commerces locaux, et répertorie les attraits naturels, culturels et historiques ainsi que des activités proposées par les Montréalais. La carte-guide est résolument «verte». En outre, c'est la première fois qu'une carte-guide touristique de la National Geographic regroupe des citations et des dessins d'enfants décrivant leur ville dans une section appelée «Une ville pour les enfants» (National Geographic Society, National Geographic et la Ville de Montréal lancent la première carte-guide géotouristique de la ville, 15 juin 2009).

Tel que mentionné précédemment, le contenu de la carteguide a été élaboré, en grande partie, grâce à la participation des résidents de l'Île-de-Montréal. De novembre 2008 à janvier 2009, les citoyens ont été invités à remplir un formulaire sur Internet pour soumettre une liste de leurs attraits préférés 
(http://www.geomontreal.com). Ce formulaire a été conçu pour mettre en valeur Montréal en demandant aux gens de désigner les sites et points d'intérêt de leur voisinage. Au cours de ces trois mois, une campagne publicitaire grand public a été lancée dans le but d'informer les citoyens sur ce projet. Plus de 400 attraits ont été répertoriés. Le comité et la National Geographic ont ensuite examiné ces suggestions pour finalement aboutir à une sélection de 102 sites qui respectaient les principes géotouristiques tout en représentant le mieux le caractère unique et distinct de Montréal. D’autres éléments ont été incorporés à la carte-guide, notamment une chronologie des événements marquants de l'histoire de Montréal, des conseils pour les géotouristes et sept éléments-thème typiques de la destination. En consultant cette carte, on comprend vite pourquoi Montréal est une destination «à voir absolument» : vie urbaine animée, multitude de festivals et d'événements culturels et sportifs, réseau de musées, et aménagements récréatifs et de plein air. Montréal est également distincte par son caractère français et par la richesse de sa mosaïque culturelle «en répondant aux critères élevés de la Charte géotouristique de la National Geographic Society, Montréal figure désormais comme un exemple en tant que ville écoresponsable et pionnière en matière de tourisme durable» (Gérald Tremblay, maire de Montréal, communiqué de presse, National Geographic et la Ville de Montréal lancent la première carte-guide géotouristique de la ville, 15 juin 2009).

Selon l'ensemble de la communauté montréalaise et du comité en géotourisme, la carte-guide s'est avérée un grand succès. Cette réalisation a bénéficié de la couverture des journaux, des réseaux de télévision, des blogues Internet et d'autres médias, et ce, dans les deux langues officielles. Cet intérêt ne s'est pas estompé au fil des mois qui ont suivi le lancement. Non seulement les journalistes ont-ils rapporté la nouvelle, mais ils ont souligné l'utilité de la carte-guide après avoir pris le temps de l'examiner.

À ce jour, la diffusion de la carte-guide a été limitée au Québec et dans les provinces voisines. 130000 exemplaires ont été imprimés. Au printemps prochain, 400000 exemplaires seront distribués aux États-Unis par le biais du magazine National Geographic Traveler.

\section{Le géotourisme et les nouvelles technologies}

Maintenant que ce nouvel outil est à la disposition du public, la prochaine étape consiste à regrouper les champs culturel, touristique et technologique. Le comité en géotourisme étudie trois projets pour l'avenir :

La refonte du site Web : Le site web http://www.geomontreal.com actuel est bilingue (français et anglais) et vise à informer. Il explique et illustre le projet géotouristique. La carte-guide et la liste d'attraits sont téléchargeables en format PDF. Il est évident que le site doit évoluer vers le Web 2.0 et proposer un contenu dynamique.

Par ailleurs, pour maintenir l'intérêt de la communauté montréalaise dans la carte-guide et pour favoriser sa participation dans de futurs projets géotouristiques, la mise en place d'une tribune d'échanges s'impose. La nouvelle plateforme utilisera une technologie de pointe de manière à pouvoir modifier ou mettre à jour le contenu de la carte-guide virtuellement. Aucun échéancier n'est encore prévu pour la refonte du site, mais des modifications, notamment l'utilisation d'un logiciel de gestion de contenu (CMS), sont prévues pour l'an 2010.

La carte-guide interactive : Une fois le site Web reconstruit, la prochaine étape sera de transformer la carte papier en version interactive. Prenant en compte les coûts élevés et l'expertise exigée, ce projet sera sans doute réalisé conjointement avec une tierce partie. Déjà Tourisme Québec et Bell Canada se sont montrés intéressés. Bien qu'aucun échéancier n'ait été établi pour la réalisation de la carte-guide interactive, le comité souhaite sa création avant 2011.

La baladodiffusion : Dès l'origine du projet de la carteguide, une étude a été menée pour évaluer la possibilité de créer un parcours guidé en baladodiffusion (podcast), répondant aux principes géotouristiques (Joly, 2009). Les résultats ont démontré que la baladodiffusion constituerait un excellent complément à la carte-guide. En effet, elle permet d'ajouter d'autres attraits et ouvre une voie «verte» à l'expérience montréalaise. La création d'un parcours géotouristique guidé en baladodiffusion renforce la notoriété de certains points d'intérêt. En d'autres mots, la baladodiffusion permettrait d'enrichir l'expérience de l'utilisateur de la carte-guide lors de sa visite de Montréal.

\section{Conclusion}

Grâce à la carte-guide, Montréal est devenue un modèle en matière de géotourisme urbain. Bien que la ville ait servi de projet-pilote, les résultats probants ont été instantanés. Ceci prouve que le concept de géotourisme peut s'appliquer à d'autres types de destinations, que ce soit un pays, une région, etc. Le géotourisme en est à ses débuts, particulièrement celui qui met l'accent sur les destinations urbaines. Certaines des plus grandes villes du monde ont les yeux tournés vers Montréal et ont déjà contacté la National Geographic Society pour adhérer à la Charte en géotourisme. En tant que ville pionnière, Montréal peut influencer l'orientation de ce nouveau concept, surtout si elle continue de demeurer à l'affût des nouvelles technologies pour mettre en valeur le géotourisme.

Note

1 Liste des dix cartes-guides géotouristiques : Sierra Nevada, côte nord de la Californie, Central Cascades, Montréal, Appalachia, Arizona/Sonora, Baja California (Basse-Californie), Crown of the Continent, Greater Yellowstone, et Vermont.

\section{Bibliographie}

LAUZON, André-Jean (2009) Le projet géotourisme Montréal, étude de cas, Université du Québec à Montréal, 47 p.

JOLY, Marie-Claude (2008) Les technologies de l'information dans la mise en valeur du territoire géotouristique montréalais, Université du Québec à Montréal, $45 \mathrm{p}$.

JOLY, Marie-Claude et Dinu BUMBARU (2007) An Application to Adhere and Implement the Geotourism Charter in Greater Montréal, Montréal.

MOFFET, Barbara (2009) National Geographic and City of Montréal Launch First Urban Geotourism MapGuide, Washington.

Center for Sustainable Destinations, <www.nationalgeographic.com/travel/ sustainable/about_geotourism.html > (Consulté le 20 août 2009)

National Geographic Society (2009) National Geographic et la Ville de Montréal lancent la première carte-guide géotouristique de la ville, 15 juin, $3 \mathrm{p}$. 